\title{
COVID-19 lockdown increased diabetic mortality, morbidity and costs in Italy
}

The COVID-19 pandemic lockdown in Italy resulted in a reduction in patient access to healthcare, an increase in diabetic-related mortality, and an increase in costs, according to the results of an analysis published in ClinicoEconomics and Outcomes Research.

The researchers examined the effects of the lockdown on specialist visit reduction, excess mortality in the diabetic population, and the economic effects of the slowdown in the use of innovative diabetic therapies. The overall impact on QOL and costs were also estimated. Data were taken from visits to a Genoa facility and published literature. The economic analysis was from the perspective of the Italian NHS.

During the 2020 COVID-19 lockdown, there was a 28\% reduction in patient access to diabetic healthcare. Diabetic patients also recorded mortality that was twice as high as the general population ( $20.4 \%$ vs $10.2 \%$, respectively). There was also a reduction in the consumption of new antidiabetic therapies $(-14 \% ; 27 \%$ vs $41 \%)$. This was estimated to cost $€ 26.6$ million for the Italian NHS, with a loss of 257 utilities in diabetic patients due to the missed benefits of access to healthcare and treatments during the lockdown. Simulation scenarios estimated the overall expenditure could range from $€ 38.7$ million to $€ 94$ million with a loss of $294-836$ utilities.

Torre E, et al. Economic Impact of COVID-19 Lockdown on Italian NHS: Focus on Diabetes Mellitus ClinicoEconomics and Outcomes Research : 1 Jul 2021. Available from: URL: https://doi.org/10.2147/CEOR.S313577 